\title{
Information systems for primary health care: the case of the Aegean islands
}

\author{
J. DARZENTAS and T. SPYROU \\ Research Laboratory of Samos, Department of Mathematics, University of the Aegean, GR 83200 Karlovassi, Samos, Greece
}

\begin{abstract}
The concern of this paper is the creation of an information system for primary health care. The problem environment is characterised by its location - health centres and agrarian surgeries serving small to medium size communities, which make up insular societies on the Aegean islands in Greece. The approach adopted was based on soft systems methodology (SSM) with the aim of obtaining operational models of the problem situation requiring the information system (IS). The SSM approach has proved most efficient in identifying important subsystems within the primary health care system. It also aided in this way the debate about the solutions to the problem of creating an IS in a health centre on the island of Samos.
\end{abstract}

\section{Introduction}

Problem identification and understanding in human activity environments is a key phase in any attempt to deal with situations requiring reorganization and/or improvement. Design and implementation of information systems (IS) in organizations are not new issues and have been tackled successfully in many cases (Symons \& Walsham, 1988; Mathiassen \& Nielsen, 1989). The considerable amount of research and development experience has raised a number of important points which require refinements or extensions to existing theories, methodologies and techniques in order to accommodate more of the 'real problem' and provide better overall solutions. Problems are usually defined in low-level terms so that they can be rigorously specified. As IS matures, researchers feel the need to tackle the higher (conceptual) problems as well, where 'hard' approaches (such as structured systems analysis, 'hard' operation research (OR) cannot be applied owing to difficulties in strictly specifying the problem.

This phenomenon has been observed in OR, for instance, where researchers and practitioners, rather than accepting 'specified problems' and solving them using 'hard' OR tools, looked back into the problem, demanding, discovering and adopting 'soft' tools and approaches for providing more representative 'suggestions' towards possible solutions. This maturing and resultant extension in the range of problem recognition

Eur. J. Inf. Systs. Vol. 2, No. 2, pp 177-127, 1993 often results in what is sometimes taken as a crisis of identity in the field in question.

In IS development this need for extension is more apparent when problem understanding at higher conceptual levels is required, where the problem is usually identified within ill-defined systems. Amongst these cases is the one presented in this paper, that of the design and implementation of an information system for a primary health care (PHC) (sub)system with the special characteristic of having been tailored to the needs of insular societies such as those of the Aegean islands in Greece. Such a subsystem, as anticipated, was found to be very difficult to capture owing to the richness of issues and views involved in its operation. The ties with the local society, the cultural and political elements which are very pronounced and the inability of the heavily centralised health authority to propose legislation that can be interpreted meaningfully by the doctors and the rest of the staff are just a few of the issues involved. In addition, the willingness to incorporate an IS into the health centre's everyday operation can be described as a 'sign of the times', without however any real understanding by the actual 'owners' of this 'desire' as to what the new technology would really do. Therefore to create an IS for the health centre, an understanding of the system and its actors are prime prerequisites. In other words, the problem is at a high conceptual level, rather than at the level of technology transfer.

The soft systems approach when applied to information systems (Wilson, 1984, 1990; Checkland \& Scholes, 1990) offers the potential for developing operational models which accommodate different perceptions of the problem at high levels. In dealing with the situation at 
these levels, there is a need to include the idiosyncratic, subjective and cultural elements necessary to define the information required, and in turn, to be able to design appropriate information systems. Thus soft systems thinking (Checkland, 1981; Checkland \& Scholes, 1990) is of primary importance here for satisfying the conceptual prerequisite of problem identification and understanding. This paper views the problem situation within a primary health care subsystem as a 'soft' ill-structured human activity subsystem, and applies soft system thinking to resolve it. This paper presents an account of how SSM successfully aided such a process in the environment of a health centre in the Aegean, which is described in the following section.

Primary health care (PHC) (an institution only recently established in Greece) arouses, both nationally and internationally, considerable social, economic and scientific interest, involving the most fundamental act of health care, the prevention of disease. The regulations governing the structure of the PHC units in Greece, known as health centres, are laid down by the Preventive and Social Medical Act. In addition to preventive medicine they are also required to accommodate the needs of urgent medical care.

The work presented here aimed at the creation of an information system for PHC in island societies, using a health centre in Samos as a test case. As already mentioned, from the first instance the human activity subsystem in question was found to be very rich operationally and structurally. Any attempt towards creating an IS would require a wide understanding of it. To this end, soft systems thinking was applied in order to identify, understand, interpret and accommodate the various relevant worldviews about the problem space and to discuss solutions at various levels working towards the implementation required. The health centre of Karlovassi town on the island of Samos was taken as representative for the work presented.

The next section briefly outlines the general framework of the National Health System (NHS), of which the PHC is a subsystem. It also describes the natural island ecosystem which sets the background for the present work. The following section presents briefly SSM and its applicability to information systems. In the subsequent section the actual application of SSM, as well as the reasons for choosing it for the design of a health information system, are presented. Finally a summary and discussions are given.

\section{The National Health System in Greece}

The World Health Organisation (WHO) specifies that PHC consists of medical care, social care and prevention, and that these are the means to achieve WHO's objective: 'health for all by the year 2000' (WHO, 1978, 1979). It defines 'health' as being not just the absence of disease or infirmity but the wider concept of complete menial, corporal and social welfare. Also 'disease' is not just a biological phenomenon but is linked to social, financial and political factors. So the solution to the problem of health maintenance and health promotion does not lie solely in medical treatment.

In Greece, this definition of health was officially adopted in 1983 when it was included in the law on the National Health System (NHS). Figure 1 shows the NHS organization's conceptual scheme and how the newly introduced PHC ideas were incorporated. What can be seen is an expansion of the old NHS organization with the addition of the health centres as decentralised organic units of the prefecture general hospitals, their brief being to provide PHC.

The complete dependence of the health centres on the prefecture hospitals in terms of organization and functioning resulted in a mechanistic transfer of the functional patterns from the second and third degree hospital-medical care (clinical, outpatient surgeries, laboratories and agrarian surgeries) to the health centres. At first, this transfer seemed to be effective and in some cases may still be convenient since it satisfies some basic medical care needs. However, it gradually became apparent that the development of PHC was being impeded, in particular the advances in the development of social care and preventive medicine for present dayneeds and in accordance with WHO directives.

During 1985, the Central Health Council reported on the various weak points in the NHS with reference to PHC and health centres' utilisation. It was acknowledged that the successful development of PHC depends to a great extent on its degree of autonomy and on the independent development of health centres, and that the existing functional and financial structure of health centres, as introduced by the law, obstructed the PHC aims and objectives. Accordingly, a number of alterations were proposed by the Central Health Council. These concerned the transfer of basic responsibilities to the health centre's director, partial financial independence and the introduction of community participation via the setting up of local health committees. The objective was for the health centres to become more selfgoverning and thus to be able to deal more realistically with the actual problems of the local community.

The specific problem environment studied here was primary health care in the Aegean island ecosystem. Geographically the zone is made up of a large number of small islands, scattered over the Aegean Sea. It is divided into five regions as shown in Figure 2.

The general hospitals and their dependent health centres are also shown in Figure 2. It can be seen that five hospitals are distributed amongst the most densely populated islands, in the capital island of each region. Patients requiring advanced (tertiary) medical care are referred to specialists in Athens. There are also twenty 


\section{National Health System}

Greece (L. $1278 / 82$ L. 1397/83)

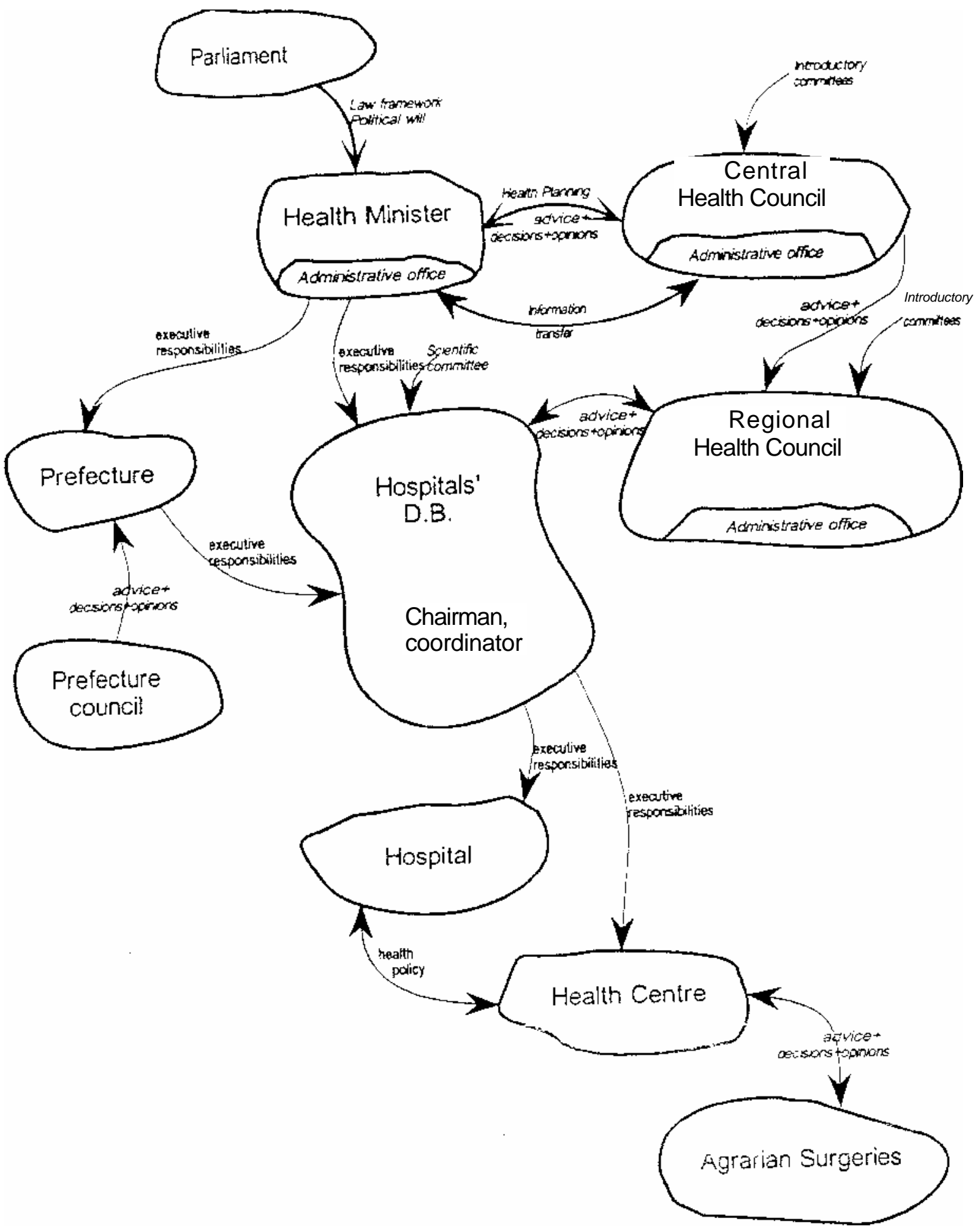

Figure 1 The National Health System in Greece. 


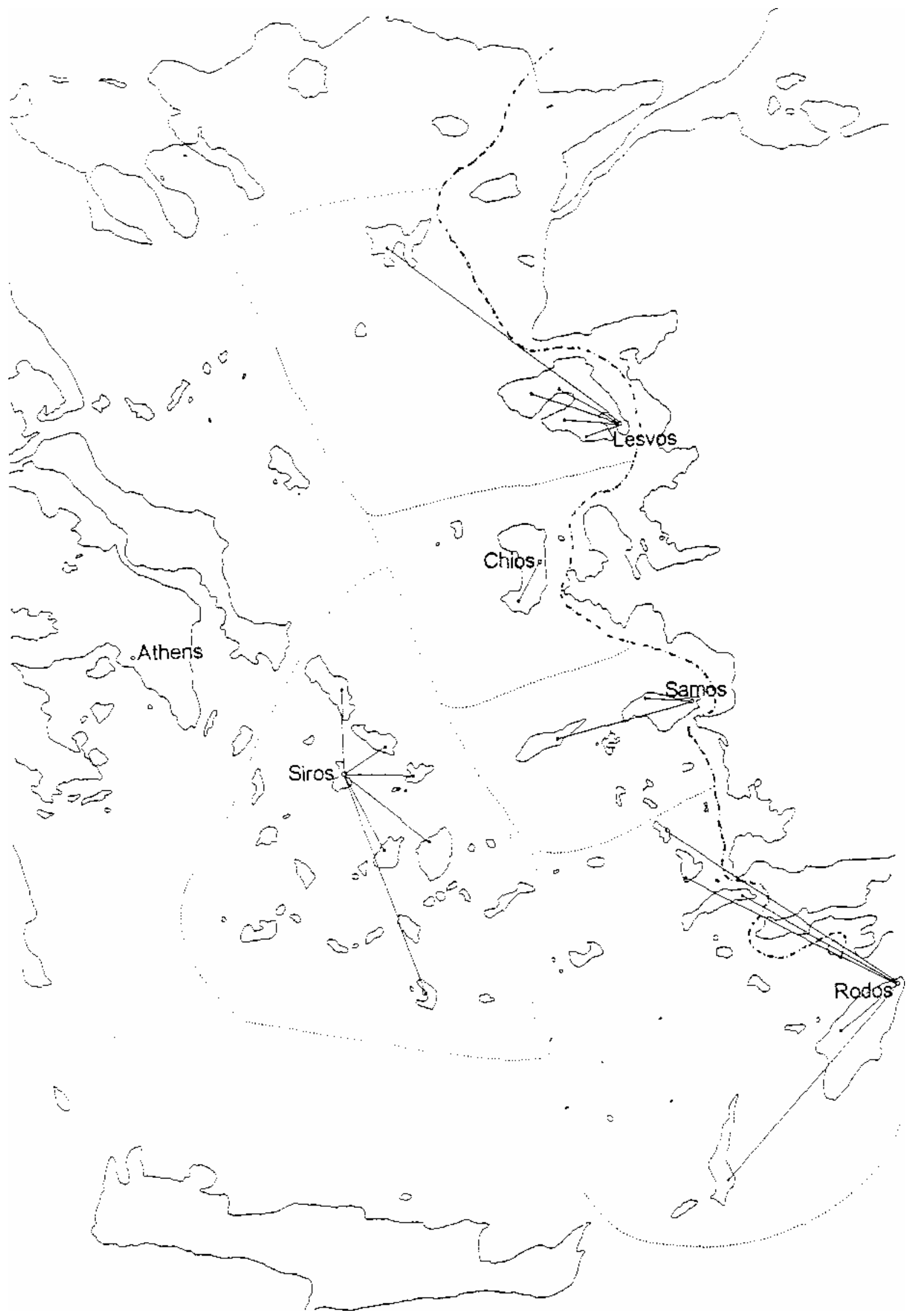

Figure 1 Geographical distribution of the General Hospitals and the health centres in the Aegean islands. 
health centres placed at key points. The locations of the general hospital in Samos town, the health centre in Karlovassi town, and its agrarian surgeries, are shown in Figure 3.

It is worth noting that since the PHC system established through the health centres is relatively new in Greece, there is an opportunity to tackle it, in terms of the introduction of information technology, as a small newly established subsystem which is more easily definable compared with the genera] health system in Greece which is ageing and inflexible.

\section{Soft systems methodology for health care}

In a conventional approach a primary objective of an information systems (IS) analyst, in order to design a formal information system, is the construction of a reliable operational model of the situation. This is usually feasible when facing 'hard', well-structured problems but not for 'soft', ill-structured, human activity systems.

Experience has shown that even well-assembled information systems for 'soft' human activity systems have collapsed because their shortcomings became apparent during their operation (Iivari, 1991). As Checkland (1985) points out: 'SSM was developed because the methodology of systems engineering, based on defining goals or objectives, simply did not work when applied to messy, ill-structured, real-world problems.'

There are various application generators (e.g. CASE tools) that have contributed to IS development even to the extent of enabling the client to play the principal role in the process of design. Nevertheless, they also show their best potential in the 'hard' problems. As a result, these tools have concentrated on the 'technical ${ }^{1}$ aspects of IS design and the tendency is for a decrease in the amount of time spent on problem diagnosis; current estimates suggest that only $20^{\wedge} \mathrm{o}$ of a project is spent upon problem diagnosis and $80^{\wedge} \mathrm{o}$ upon the design itself (Stowell et. al., 1990).

On the other hand, where improved problem understanding must be sought, which is the norm in soft, illstructured problem environments, such as health care systems, SSM and systems thinking generally has been a popular choice \{Checkland, 1987; Watson \& Smith, 1988; Stowell el at., 1990). This is because messy, soft problems are often encountered in information processes in medical practice. These information processes can be divided into two categories (Furukawa, 1990). One relates to information utilisation and the circulation process between specialised activities. This category could to some extent be served by a typical 'hard' systems analysis methodology. The other category, however, is directly related to diagnosis and/or therapy, which both concern a physician's mental activities. The information processes corresponding to the latter category are based on observation and on the application of the physician's knowledge as a result of the patient-doctor relationship. It is this process which generates the medical-health information. To take into account this type of information a methodology which can accommodate its characteristics should be employed. Thus, SSM was chosen, rather than traditional 'hard' systems thinking.

A crucial distinction has to be made between systems thinking and real-world acting, a key distinction in this methodology. Systems thinking activities are those

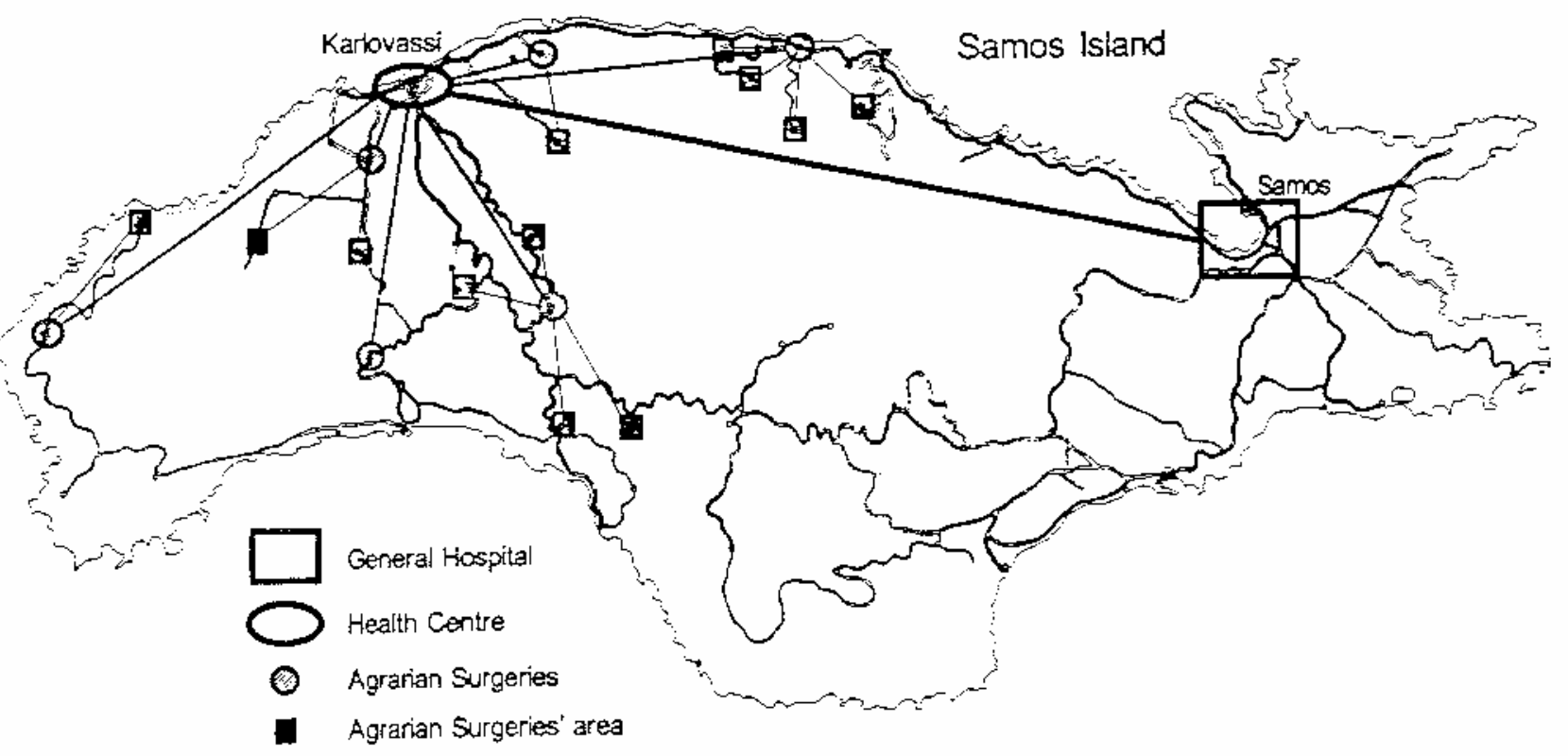

Figure 3 General Hospital, heajth centre and agrarian surgeries in west Samos.

Figure 3 General Hospital, health centre and agrarian surgeries in west Samos. 
activities which allow thinking about the real world in systems terms, namely the formulation of relevant root definitions and the building of corresponding conceptual models, and these come before and after real-world activities. In this way SSM maintains the important difference between thinking about and being, between the real world and thinking about the real world.

Using the methodology within its systems thinking process, the analyst has to formulate root definitions, to assemble the minimum amount of necessary activities and to structure activities into conceptual models. Here the use of several relevant systems is suggested in order to stress the different viewpoints and beliefs of the actors involved, and thus express the subjective nature of SSM. SSM accommodates different interests and initiates debate. This accommodation is represented by the systemically desirable and culturally feasible proposed changes. The debate about changes is structured by the comparison of the conceptual models against the participants' perceptions of the part of the real world under examination.

\section{Application of an approach based on soft systems methodology}

The problem owners, the instigators of the project, were an active subgroup of the health centre's staff, consisting primarily of doctors, who wanted changes to the health centre and felt that IS would be an appropriate vehicle. The membership of the subgroup was mostly doctors of local origin with vested interests in developing strong PHC in the area, although without definite views or clear understanding of what IS could offer to achieve this aim.

To apply an SSM based approach for understanding and formulating the problem situation of the health centre system, a large number of interview/discussion hours took place, in an effort to extract the different views about the situation. Interviews were held with the whole staff of the health centre in Karlovassi town, consisting of the director, three general practitioners, four field doctors, two dentists, six nurses and related staff (such as secretaries, receptionists and clerks), in order to yield a 'rich picture' (Checkland \& Scholes, 1990; Stowell et at., 1990) concerning the real-world problem situation. The interviews were unstructured so as to reveal any possible beliefs and personal feelings about the different world views.

The core themes around which these interview's and discussions revolved can be summarised as follows: problematic areas in professional sections; issues that produced and/or contributed to this problem; others sharing the same problem; the urgency to solve this problem and why; possible solutions; feasible solutions; parts of broader problems; opinions about a concern with wider problems instead of specific ones; restrictions to specific situations; other areas of concern for the analyst. One rich picture which resulted from the above is shown in Figure 4.

Some factors (identified during the 'finding out' phase of SSM) which affect the problem/system in the case of the island populations include:

- The lack of tertiary health care (university hospitals) and specialised secondary health care (general hospitals). This leads to patients moving from the islands to the big cities. This causes disruption in the flow of health information between the various classes of health care. It is left to the patients to inform the local health system about their health situation.

- The existence of a seasonal increase in population in the holiday season which disrupts any preventive or training programme during the four summer months.

- There is a well-defined and stable community during the remaining eight months with no remarkable changes in life-style. The family doctor tends just to remember the greater part of a patient's medical history.

- The particular geographical conditions, for example inaccessible areas, impede the contact between doc tors and patients, especially in the agricultural com munities, even though there may be serious health problems. Furthermore other factors such as negative patient attitudes toward the doctor, owing to the lack of health education and also the desire to preserve privacy in these tightly knit communities, make would-be patients think twice about consulting the local doctors.

- Climatic and regional conditions result in a limited variety of occupations, which imposes a certain style and method in approaching, informing and treating the population.

- Private health-care exists on the islands but is not well established, which means that the information loss to this sector is negligible.

As expected, the use of SSM identified vital elements of the system's behaviour in relation to the medical activities for which the information system was created. These include the cultural characteristics of the staff as a whole,, which are embedded in the root definitions presented, and discussed below and which otherwise would not have been identified. Several root definitions (RDs) of the health centre were suggested stemming from the world views:

$\mathrm{RD}_{1}$ : A medical treatment organization. part of the national health system.

$\mathrm{RD}_{2}$ : A health care organization for areas where there are no other organized health care services offering medical treatment to the people of the area.

$\mathrm{RD}_{3}$ : The national organization of an area, responsible for the health of the people of this area.

$\mathrm{RD}_{4}$ : A way to give motivation to specialised doctors of the private sector to shift to the national health 


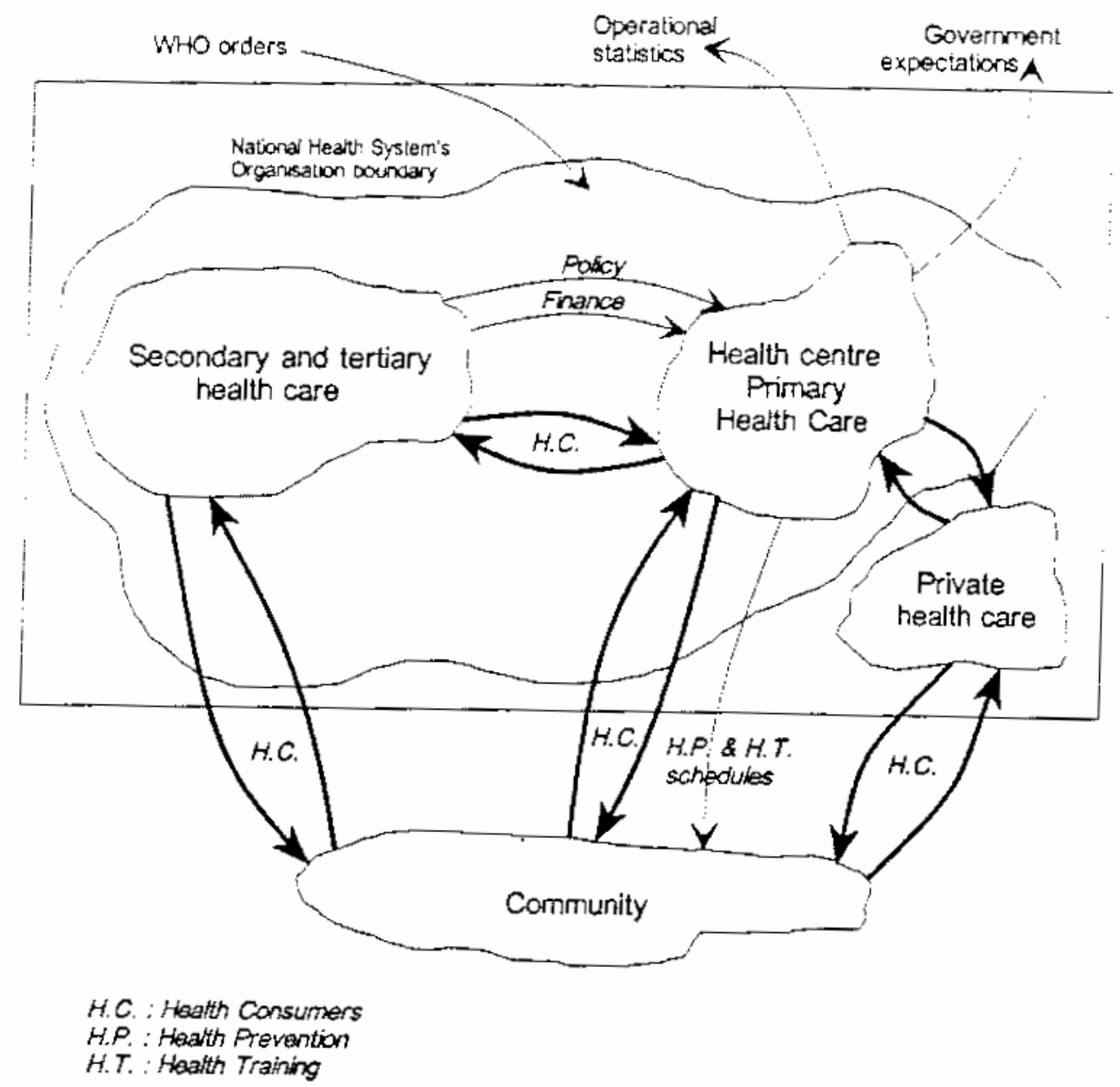

Figure 4 A rich picture of the Health System.

system in areas where no health care is provided.

$\mathrm{RD}_{5}$ : Free medical care.

$\mathrm{RD}_{6}$ : The governmental attempt to establish public medical services in areas where private medicine is not well developed.

$\mathrm{RD}_{7}$ : A unit of the national health system whose job is to provide medical and emergency treatment, as well as preventive care to the people of an area.

Various cultural characteristics are reflected in the significant differences amongst the root definitions such as:

- Different understandings of the health centre's aim, i.e. medical treatment versus health care. This is mainly due to the existence of medical staff who have learned to operate in the hospital environment and do not wish to change.

- A tendency to present a negative attitude sometimes for political reasons.

- Support of private versus public health care and vice versa for economic and political reasons.

- Some see the health centres as part of a rural develop ment policy, while others see it as an expansion of the philosophy and practice of PHC.
The root definition of the health centre's PHC subsystem identified and agreed upon by the problem owners after some debate as the most relevant at this stage, was: 'Local structure of primary health care providing medical care and preventive medicine and training for the population and the visitors of an area operating as a decentralised unit of a general hospital.'

Figure 5 shows the corresponding conceptual model of the PHC. That conceptual model was stabilised by the process of discussions/interviews mentioned above, i.e. making changes in the root definitions and the corresponding conceptual models. The same procedure was followed at every level in this approach.

At the resolution level corresponding to the above conceptual model, there are three identified subsystems (Figure 5):

1. The preventive medicine and health training subsystem which actually designs, executes and monitors plans for health training and prevention; for instance, training in diet and nutrition in the case of fishing communities. In the same communities, the long-term effects of particular diets may also be studied.

2. The PHC 'consumer' processing subsystem dealing 


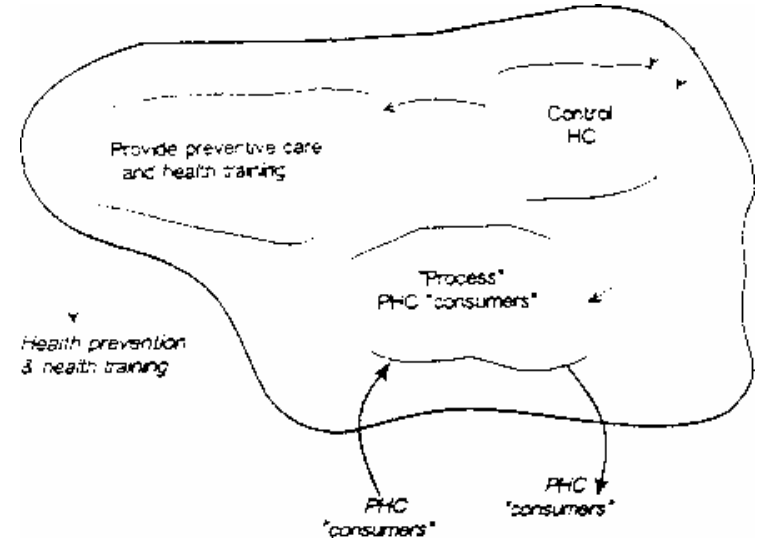

Figure 5 First level conceptual model of the health centre system. with patients needing medical care, emergency treatment or to undergo preventive medicine programmes. 3. The health centre control subsystem which formally controls the operation of the whole health centre.

The next level leads to the conceptual model shown in Figure 6. The three subsystems are described in more detail via relevant identified activities. The activities are at an operational level where the characteristics and imposed peculiarities of the information system begin to take form.

In this case, the application of SSM as an interpretive learning approach made it possible to gain new insights into real-world activities through their comparison with those that are desirable in systems terms. In other words, it was accepted by the problem owners and, by and large, by the rest of the staff that the main purpose of the IS for PHC at Karlovassi was primarily expressed by subsystem (1) (a totally new and very important one in terms of information systems planning) backed by subsystems (2) and (3). This outcome was different from that originally expected by all those involved including the problem owners. SSM focused the health centre systems analysis in relation to implementation issues as well as clarifying the context for the development of IS components.

The purpose of subsystem (1) is to monitor and train the population in relation to health matters using prevention plans, as well as to intervene when deemed necessary. Subsystems (2) and (3) relate informationally to subsystem (I) in terms of possessing and processing information relevant to the purpose of subsystem (1).

The prevention plans are continuously altering, depending on the type and magnitude of planned prevention. These changes depend on feedback from, for example, pilot studies, as a result of which the type and nature of pans of these plans may have to change.

During the comparison phase between the conceptual model of subsystem (2) and the real situation, it was found that a mechanistic transfer of operational practices existed from the other levels of health care to the PHC. In these other levels (mainly hospital based) there exist well-established information structures, such as the medical records on paper with specific operational destinations and specific requirements, this being the standard act of medical care. That in fact means that some more general structure of the information requirements of each activity as well as its informational output will be known and expected. However, it was found that the whole patient medical record history was not needed for the operation of subsystems (1) and (2) as had previously been thought by the health centre's medical and administration staff at the beginning of the project. There is an immediately identifiable dependency between subsystems (1) and (2), since the information subsystems which serve them aim to maintain a flow of information in order to serve and protect patients' health, while an information system designed for subsystem (3) will only aim to maintain the information flowin order to control and manage the health centre.

Subsystems (2) and (3) would have been closer to what the 'customer' originally had in mind for an IS: in other words, information systems dedicated to serving subsystem (3) and to the maintenance of integrated personal medical records. However, apart from subsystem (1), which was a new realisation and which requires flexibility in information management, subsystem (2) was also found to be different in operational terms from that expected, i.e. different from a product of the mechanistic transfer from the other levels of medical care. This is because its informational requirements should correspond to those of subsystem (1).

All these factors mentioned above indicated the need for changes in the consideration of the approach to be used for implementing a PHC IS. To this end the notion of a flexible medical record shell in place of the personal medical record was adopted in order to satisfy the peculiarities of subsystem (1). This shell provides the kind of environment that allows (and dictates) the personalisation of the informational structuring according to the special characteristics of the prevention plans utilised by each practitioner. This allows for instances of medical records to be formed by the prevention plan maker and offers the potential to transform information elements according to requirements.

For instance, the paediatric department of the health centre planned a prevention activity in order to investigate, describe and intervene in the immunisation programme of the infant population of the area it covers. The flexible medical record shell was formed according to the initial information needs of the planned preventive action. This record was very soon after (and at least twice) altered to accommodate a set of parameters which were deemed necessary after the action was 


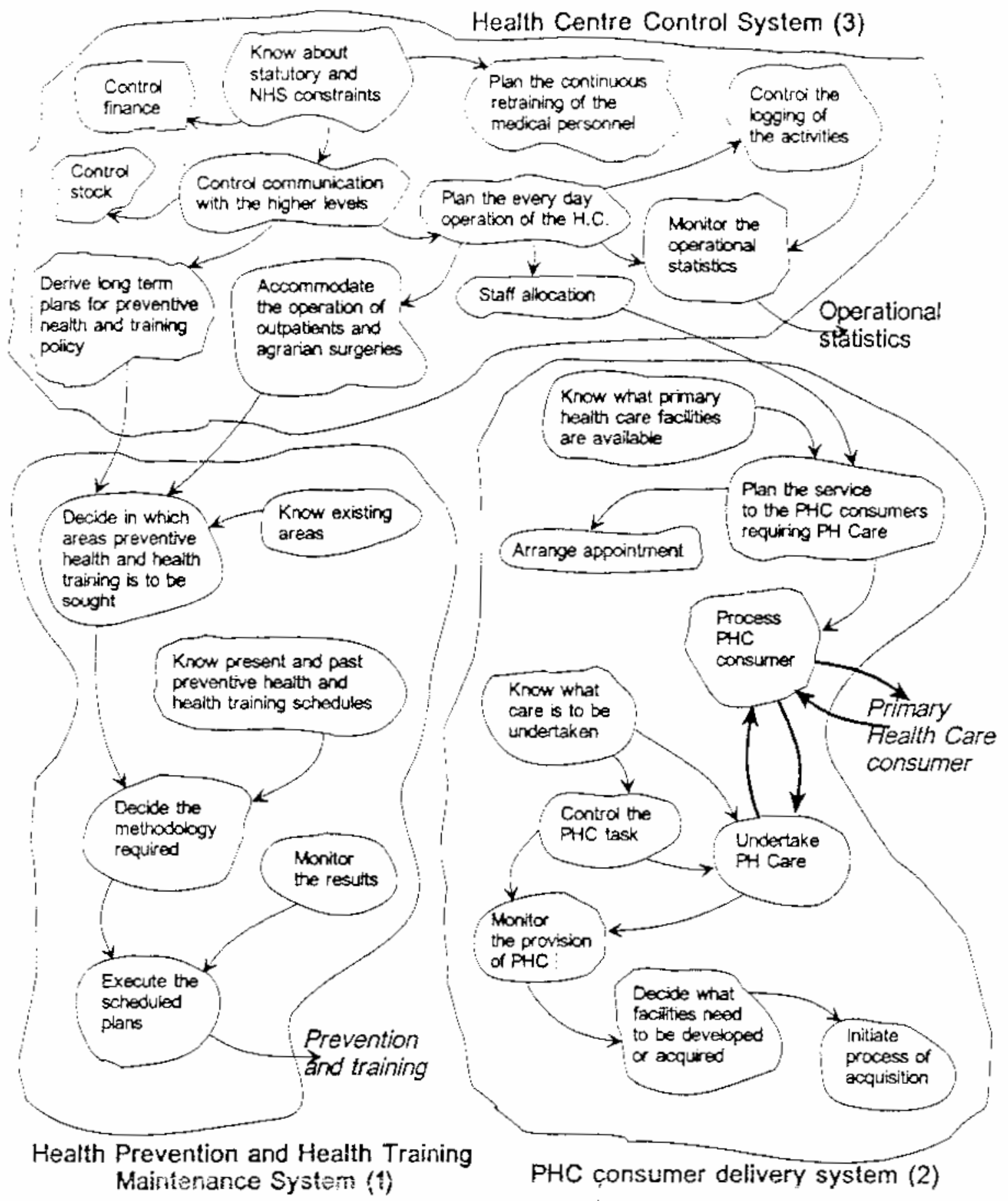

Figure 6 Second level conceptual model of the health centre system.

initiated and were due to the need, in this case, for intervention.

This 'attitude' towards implementation which is based on the flexible shell was due to the use of SSM which was the main reason for recognising subsystem (1).

An example of the required flexibility is the following. Within three years the dental care offered at the health centre of Karlovassi changed from that imposed by the initial law, which was caring for children up to 18 years of age, and was extended to caring for the elderly as well as everyone in emergencies; and ended up making appointments for everyone. This was a result of political and social pressure, primarily by the local community. The informational requirements and output of the activities determined at the next levels of analysis (Figure 6) (through the application of SSM) offer information categories embedded in information flows, within which the requirements of the prevention planner are expected 
to be contained, hence a medical record for this purpose could be made. Figures 7 and 8 present, as an example, a small section of the conceptual models of subsystem (2) and (1) respectively at the next level of resolution to the one shown in Figure 6. The informational requirements and output of the activities shown in these figures, routinely identified at this stage, provided all the necessary components for the actual implementation.

The introduction of the shell as a notion poses two main problems in the realisation of the IS: (a) the creation and (b) the operation of the system. Relational database management systems (RDBMS) technology was chosen to tackle the first and continuous training the latter.

\section{Summary and conclusions}

In this paper, a project aimed at the creation of an information system for a health centre - a primary health care subsystem - is described. The health centre offering preventive and emergency care is situated on the island of Samos in Greece and has a considerable catchment area. It officially belongs to the island's hospital. The problem environment, as expected, proved to be a complex one reflecting an ill-defined, human activity system. The problem owners were an active group consisting mainly of doctors who wanted changes via IS technology. The actors involved consisted of all the medical and related staff of the health centre.

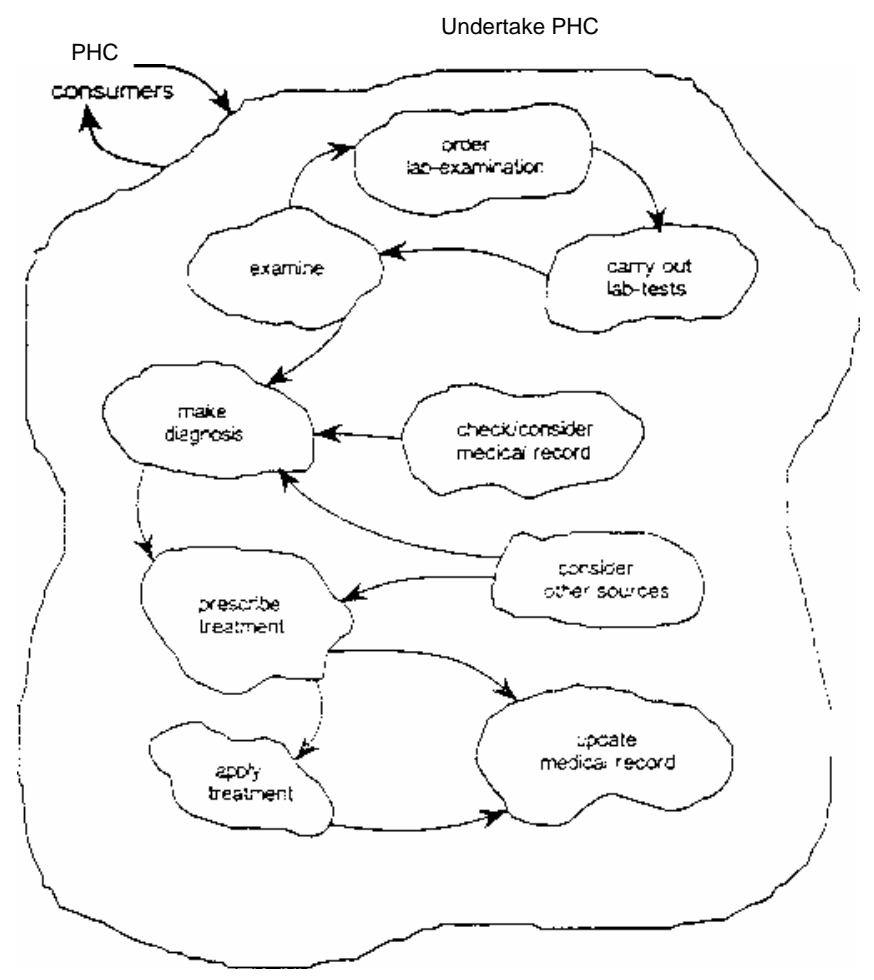

Figure 7 Third level conceptual model of the 'Undertake PHC' activity.

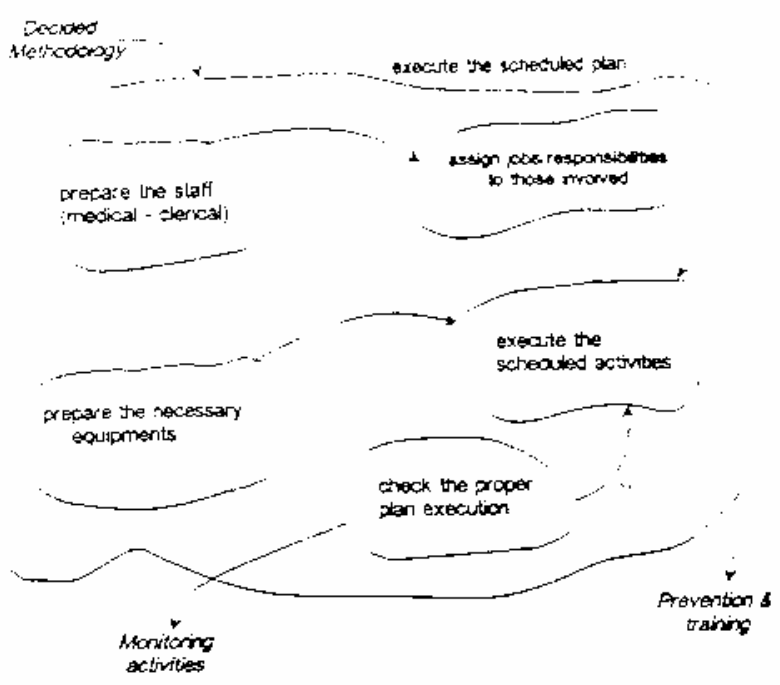

Figure 8 Third level conceptual model of the "Execute the scheduled plan' activity.

The approach adopted was based on the soft systems methodology in order to learn about and understand the problem situation requiring the IS, with the aim of putting forward models which accommodate the different views and perceptions of the actors involved. These models were used in the debate about the form of the IS required. Even though SSM was not developed primarily for use in the development of information systems, it has proved very efficient in this case.

When applied to the problem described in this paper - an IS for a PHC system in the Aegean islands, especially the health centre in Karlovassi, Samos - SSM helped to uncover a new and more representative understanding of real-world activities in relation to what is desirable in systems terms. In this case, it became apparent that the IS for the health centre in Karlovassi offering PHC was expressed by subsystem (1): a subsystem which concerns the training and monitoring of the population in preventive care. This was not originally conceived by the owners of the problem and the actors involved as the focus of the IS, and its emergence as an issue of importance is due to the application of SSM. A further point was that the use of the whole of the patients' medical record, which the staff initially believed necessary for the operation of the subsystems did not, in fact, hold true and this was realised by them.

Another important realisation due to the use of SSM was the need to introduce the notion of a flexible shell to create the personal medical information environment which was developed with the current relational database management system (RDBMS) technology.

One of the major problems encountered during the information system development and application, apart from the health centre staff having to adapt to the new, 'exotic', unknown and 'mysterious' information technol- 
ogy, was the training needs of she staff involved. To face this difficulty, training has been scheduled to continue over the whole implementation cycle. The plan is to cover all the needs of the information system end users who are unfamiliar with information technology.

Acknowledgements - This research was carried out as part of a project funded by the National Drug Organisation $|\mathrm{EOF}|$.

\section{References}

CHECKLAND P B (19S1) Systems Thinking Systems Practice. Chichester.

CHECKLAND P B (1985) From optimising to learning: a development of systems thinking for the 1990s. Journal of the Operational Research Society 36(9). 757-767.

CHECKLAND P B (1987) A note on the use of systems thinking in the provision of health care. Journal of Applied Systems Analysis 14, 129-130.

CHECKLAND P B and SCHOLES J (1990) Soft Systems Methodology in Action. Wiley, Chichester.

FURUKAWA T (1990) AI in medicine; a Japanese perspective. A! \& Society 4, 196-213,

IIVARI $J$ (1991) A paradigmatic analysis of contemporary schools of IS development. European Journal of Information Systems 1(4), 249-272.

MATHIASSEN L and NIELSEN A (1989) Soft systems and bard contradictions - approaching the reality of information systems in organisations. Journal of Applied Systems Analysis 16, 75-88.
StOWEll F A, HOLLAND P, MULLER P and PRIOR R (1990) Applications of SSM in information system design: some reflections. Journal of Applied Systems Analysts 17. 63-130.

SYMONS V and WALSHAM G (!9S8) The evaluation of information systems: a critique. Journal of Applied Systems Analysis 15, 119132.

WATSON R and SMITH R (19SS) Applications of [he Lancaster soft systems methodology in Australia, Journal of Applied Systems Analysis 15, 3-26.

WHO (1978) Primary Health Cart: Report of the International Conference on Primary Health Care, Alma-Ata, USSR, 6 December. WHO, Geneva.

WHO (1979) Formulating Strategies for Health for All by the Year 2.000. WHO, Geneva.

WILSON B (1984) Systems; Concepts, Methodologies and Applications. Wiley, Chichester.

WILSON B (1990) Systems, Concepts, Methodologies and Applications. Wiley, Chichester. 